\title{
Development of Biofunctional Textiles by the Application of Resveratrol to Cotton, Bamboo, and Silk
}

\author{
Eva Pinho*, Mariana Henriques ${ }^{1}$, Rosário Oliveira, Alberto Dias ${ }^{1}$, and Graça Soares ${ }^{2}$ \\ IBB-Institute for Biotechonology and Bioengineering, Centre for Biological Engineering, University of Minho, \\ Campus de Gualtar 4710-057, Braga, Portugal \\ ${ }^{1}$ CITAB-Centre for the Research and Technology of Agro-Environmental and Biological Sciences, Department of Biology, \\ University of Minho, Campus de Gualtar 4710-057, Braga, Portugal \\ ${ }^{2}$ Departament of Textile Engineering, University of Minho, Campus de Azurem 4800-058, Guimaraes, Portugal
}

(Received June 19, 2009; Revised September 9, 2009; Accepted October 4, 2009)

\begin{abstract}
The goal of this work was to create a new generation of greener fabrics made of natural materials. For that, resveratrol (Res), obtained from Polygonum cuspidatum extract and known to have antibacterial, antifungal, and anti-inflammatory activity, was applied by an exhaustion method to cotton, bamboo, and silk knit fabrics. The fabrics adsorption behavior was tested and the amount of Res adsorbed was determined by its decrease on the immersion solutions with time and measured by spectrophotometry at $350 \mathrm{~nm}$. The maximum adsorption capacity was observed for silk and it was independent of $\mathrm{pH}$ conditions used ( $50.5 \%$ at $\mathrm{pH}=7$ and $58.3 \%$ at $\mathrm{pH}=5$ of the initial Res concentration). At acidic $\mathrm{pH}$ conditions, cotton adsorbed $51.2 \%$ of Res and Bamboo adsorbed only $28.1 \%$ in $15 \mathrm{~min}$. However, neither cotton nor bamboo adsorbed $\mathrm{Res}$ at $\mathrm{pH}=7$. The release behavior was also analyzed and the highest Res release was observed for cotton in alkaline sweat and urine mimic solutions. The lowest release was achieved by cotton in water $(1.0 \mathrm{ng} / \mathrm{m} l)$. Moreover, no relation was found between the amounts of Res adsorbed or released and cell viability. In conclusion, this work shows that it is possible to obtain cotton, bamboo, and silk functionalized with resveratrol. The incorporating process here described is simple and silk-Res can be presented as a good combination.
\end{abstract}

Keywords: Biofunctional fabrics, Resveratrol, Antinflammatory, Antioxidant, Exhaustion process

\section{Introduction}

To regain competitiveness, textile industries have focused their research on the development of multi-functional fabrics with high added value and applications in different areas. Biofunctional textiles include antimicrobial products, obtained using special finishing applications or fiber modifications, as well as textiles with cyclodextrin incorporation, which can enclose substances or deliver drugs "devices" directed to the skin [1].

Currently, most of the antimicrobial fabrics commercialized are produced incorporating metals like silver and zinc [2]. However, they have low biocompatibility and are dangerous for the environment.

The use of natural extracts in natural fabrics might make biofunctional textiles more biocompatible and consequently, with more applications on the health field. Another advantage is to turn these textiles more environmental friendly.

Natural fibers are available in abundance, biodegradable, and renewable resources in opposition to synthetic fibers [3]. Cotton and bamboo are both cellulosic fibers. Cellulose is a biological and functional material, made of glucose with $\beta$ 1,4-glucoside bonds and has more than ten thousand of these D-glucopyranose sections without branching. Chemical and physical properties of cellulose involve not only the strength

*Corresponding author: evapinho@deb.uminho.pt and elasticity of the fiber, but also water affinity (due to its many hydroxyl groups) and permeability [4]. Cotton is made of cellulose molecules organized in fibers and its physical and chemical properties, as well as its low cost, make the textile with more importance and utility, in our days [5]. Nevertheless, bamboo has properties that are more interesting. In fact, bamboo fibers are made of cellulose in a ligneous matrix with a unique structure, unidirectional fiberreinforced composite with many nodes along its length [3]. Bamboo has antibacterial and antifungal effect and its production is ecologic and of low cost [6].

Additionally, silk is from animal source and constituted by proteins. Silk fibers are composed by high molecular weight organic polymers, characterized by repetitive hydrophobic and hydrophilic peptide sequences, similarly to human hair [7]. This similarity confers biocompatibility to silk and consequently enhances its ability to be used as biomaterials [8].

Resveratrol is a natural polyphenol, which can be found in grapes, red wine, peanuts, mulberries, and other fruits and is known to possess pharmacological activities [9]. Resveratrol is produced by plants in stress situations and has antibacterial, antifungal and anti-inflammatory, and antioxidant activities [10-12]. The traditional Japanese and Chinese folk medicine recognized resveratrol, extracted from the root of Polygonum cuspidatum, as capable of treating liver, circulatory, and skin diseases [13,14]. Moreover, topical application of resveratrol has been shown to have strong anti-proliferative and chemopreventive properties against skin carcinogenesis 
as well as protection against skin damage from ultraviolet $\mathrm{B}$ exposure [11].

The purpose of this study was to incorporate the resveratrol extracted from Polygonum cuspidatum in natural textiles (cotton, bamboo, and silk) to create greener, biofunctional, and biocompatible fabrics.

\section{Material and Methods}

A purified resveratrol extract (Res) was supplied by Wuxi Gorunjie Technology Co., China. The cotton (Jersey knit, $180 \mathrm{~g} / \mathrm{m}^{2}$, yarn linear 40/1, loop length $0.3 \mathrm{~cm}$ ) fabric was obtained from Lameirinho, SA (Portugal). Silk (Jersey knit, $170 \mathrm{~g} / \mathrm{m}^{2}$, yarn linear $120 / 2$, loop length $0.3 \mathrm{~cm}$ ) and bamboo (Jersey knit, $140 \mathrm{~g} / \mathrm{m}^{2}$, yarn linear 40/1, loop length $0.3 \mathrm{~cm}$ ) were supplied by Tebe (Portugal).

\section{Identification and Quantification of the Compounds Present on Res}

Res solution $(1 \mathrm{mg} / \mathrm{m} /)$ was prepared by dissolving $500 \mu \mathrm{g}$ of Res in $500 \mu l$ methanol $(90 \%)$ followed by filtration (nylon $0.2 \mu \mathrm{m}$, Lida, USA). The solution was diluted (1:5, 1:10, $1: 15$, and $1: 20$ ) and analyzed by high performance liquid chromatography (HPLC-DAD) on a Beckman Gold (USA) system, comprising a 116 pump, a 168 DAD detector, and a injection valve 7125 with loop $20 \mu l$ (Rheodyne, EUA). The separations were carried out in a LichroCart RP18 endcapped supersphere $(250 \times 4 \mathrm{~mm}$ I.D.; $4 \mu \mathrm{m})$ column (Merck, Darmstadt, Germany) with a pre-column of the same material. In the mobile phase, the eluent A was water/ formic acid (95:5) solution and the eluent B was a methanol solution. The eluent gradient was continuous, starting at $10 \%$ of B and increasing to $90 \%$ for $60 \mathrm{~min}$. The data of all peaks were accumulated in a range of 200 600 nm and the chromatograms were recorded at 280,320 , and $350 \mathrm{~nm}$. The data were processed by a computer equipped with software Nouveaux Gold (Beckamn, USA) and the compounds identified based on their UV-Vis spectra. The amount of resveratrol on the Res was determined by the external standard method, using pure resveratrol (Sigma, Barcelona) as standard.

\section{Adsorption Studies}

The extract was added to the fabrics following the exhaustion process and the amount of extract adsorbed at the equilibrium was determined according to the method used by Takagai and co-workers [4], with some modifications. Res (Takagai and co-workers used a $5 \times 10^{-5} \mathrm{~mol} / l$ in $25 \mathrm{~m} l$ solution) was dissolved in methanol/ $/ \mathrm{Na}_{2} \mathrm{HPO}_{4}-\mathrm{NaH}_{2} \mathrm{PO}_{4}$ buffer solution $(1: 1)$ at selected $\mathrm{pH}(5$ or 7$)$ to a final concentration of $\left(1.4 \times 10^{-3} \mathrm{~mol} / l\right)$. Samples of $0.2 \mathrm{~g}$, of each fabric (cotton, bamboo, or silk) were immersed in the solution and shaken for $60 \mathrm{~min}$, at room temperature. Samples from the solution were taken every $5 \mathrm{~min}$ and their adsorption profiles were determined by absorbance spectroscopy (350 to $700 \mathrm{~nm}$ ).

The amount of extract adsorbed by the fabrics was calculated as the difference of the mass of Res added to the solution and the mass present in the solution at the equilibrium.

After incorporation of Res, fabrics were washed with tap water for $5 \mathrm{~min}$ and dried at room temperature. Then, they were analyzed by Fourier Transform infrared ATR spectroscopy (FTIR-ATR) and by reflectance spectrophotometry. The fixation of Res on the fibers was evaluated by $K / S$ values determination, according to Kubelka-Munk's law [15].

All assays were controlled using unmodified fabrics, which were analyzed in similar experimental conditions.

\section{Controlled Release}

The amount of Res released from the fabrics, obtained after 60 min of immersion in Res $\mathrm{pH} 5$ solutions (previous section), was measured using conditions similar to the human body fluids. Three solutions were prepared as described in ISO standard test 9073-8 (2000), ISC standard test 105 E04 (1997), and ISO standard test 114948-1 (1996) [16-18]: urine, alkaline sweat $(\mathrm{pH}=8)$, and acid sweat $(\mathrm{pH}=5.5)$, respectively. The urine solution was prepared with $9 \mathrm{~g} / l$ of $\mathrm{NaCl}$ in distilled water; a solution similar to alkaline sweat was prepared with $0.5 \mathrm{~g} / l$ of hydrated-9histidine-9-hydrochloride, $5 \mathrm{~g} / l$ of $\mathrm{NaCl}$, and $6 \mathrm{~g} / l$ of $\mathrm{NaH}_{2} \mathrm{PO}_{4} \cdot 12 \mathrm{H}_{2} \mathrm{O}$ in distilled water and the acid sweat was prepared with $0.5 \mathrm{~g} / l$ hydrated-9-histidine-9hydrichloride, $5 \mathrm{~g} / l$ of $\mathrm{NaCl}$, and $2.2 \mathrm{~g} / l$ of $\mathrm{NaH}_{2} \mathrm{PO}_{4} \cdot 2 \mathrm{H}_{2} \mathrm{O}$ in distilled water.

Cotton samples with Res adsorbed were immersed in 45 $\mathrm{mL}$ of urine, alkaline sweat, acid sweat like solutions, and water. The solutions containing the fabrics were shaken and maintained at room temperature $\left( \pm 20{ }^{\circ} \mathrm{C}\right)$ and were sampled at $0,2,4,6,10,15,20,30$, and $60 \mathrm{~min}$. Res concentration was measured by spectrophotometry at its maximum absorbance wavelength $(350 \mathrm{~nm})$, using a calibration curve. Bamboo and silk were assayed following a similar protocol.

It should be noted that the complete protocol was executed at room temperature to preserve the pharmacologic properties of the extracts and the solutions were shaken to maximize the adsorption of the extract by fabrics [4]. All assays were made in triplicate.

\section{Cell Viability Determination}

Before cytotoxicity evaluation, the fabrics were sterilized at $1 \mathrm{~atm}$ and $121{ }^{\circ} \mathrm{C}$ for $15 \mathrm{~min}$.

Fibroblast 3 T3 (CCL 163) from American Type Culture Collection were used in this study. Cells were cultured in Dulbecco's modified Eagle's medium (DMEM) supplemented with $10 \%$ of foetal bovine serum and $1 \%$ penicillin/ streptomycin at $37^{\circ} \mathrm{C}, 5 \% \mathrm{CO}_{2}$. After achieving the confluence, cells were passed at the density of $1 \times 10^{5} \mathrm{cells} / \mathrm{m} l$, using trypsin. 
Cells were seeded at the density of $5 \times 10^{5}$ cells $/ \mathrm{ml}$ (six well plate) in $1 \mathrm{~m} l$ of DMEM complete medium. After $24 \mathrm{~h}$, the medium was replaced with $2 \mathrm{~m} l$ of fresh medium, the sterilized fabrics were added and incubated at $37{ }^{\circ} \mathrm{C}$ and $5 \%$ $\mathrm{CO}_{2}$. After $2 \mathrm{~h}$, the medium was removed and a mixture of $20 \mu l$ of MTS [3-(4,5-carboxymethoxyphenyl)-2-(4-sulfophenyl)2H-tetrazolium] (Promega) and $1 \mathrm{~m} l$ of DMEM without phenol was added to each well. After $3 \mathrm{~h}$, the absorbance value was measured at $490 \mathrm{~nm}$ and the results were expressed as percentage of dead cells (\%), using the number of cells grown on wells without fabrics (controls).

\section{Results and Discussion}

\section{Functionalization of Textile Materials}

Resveratrol is a natural polyphenol with a chemical structure 3,5,4'-trihydroxystilbene with cis-trans isomers $[4,9]$. The phenolic groups are responsible for the reacting capacity of Res with free radicals in order to form a stable molecule, less toxic than the original radical confirming its interesting bioactivity [13]. The plant extract used had, mainly, cis-resveratrol (78.5\%), a minor amount of transresveratrol $(12 \%)$, and non-identified stilbenes (less than $5 \%$ ) as assessed by HPLC. The error associated with the HPLC method was less than $3 \%$.

This resveratrol extract (Res) was incorporated into the fabrics by an exhaustion process. The efficiency of this method can be correlated with the extract affinity for the textile material, which depends on the chemical structure and geometry of the molecules on the extract and on the fabric [19].

Res adsorption was tested using an acidic $(\mathrm{pH}=5)$ and neutral $(\mathrm{pH}=7)$ environment which were chosen based on the extract stability and the possibility of its chemical groups to ionize. The extent of ionization influences the extract capacity to establish electrostatic and ionic interactions with the textile fibers and, consequently, influences the adsorption process and the subsequent stability of Res into the fibers [19].

The amount of Res adsorbed by the different fabrics and the time needed to achieve the adsorption equilibrium are presented in Table 1.

The fabrics (adsorbent) and Res (adsorbate) possess

Table 1. The percentage of Res adsorbed and the equilibrium time (where the adsorption was maximum) when adsorbed in cotton, bamboo, and silk, at two $\mathrm{pH}$ values of (5 and 7). [1.3 $\times 10^{-6} \mathrm{M}$ extract $]$

\begin{tabular}{cccccc}
\hline \multicolumn{3}{c}{$\mathrm{pH}^{2} 5$} & & \multicolumn{2}{c}{$\mathrm{pH}=7$} \\
\cline { 1 - 2 } \cline { 5 - 6 } & $\begin{array}{c}\text { Res adsorbed } \\
(\%)\end{array}$ & $\begin{array}{c}\text { Time } \\
(\mathrm{min})\end{array}$ & & $\begin{array}{c}\text { Res adsorbed } \\
(\%)\end{array}$ & $\begin{array}{c}\text { Time } \\
(\mathrm{min})\end{array}$ \\
\hline Cotton & $51.2 \pm 0.5$ & 15 & & $0.0 \pm 0.0$ & - \\
Bamboo & $28.1 \pm 0.2$ & 15 & & $0.0 \pm 0.0$ & - \\
Silk & $58.3 \pm 0.1$ & 20 & & $50.5 \pm 0.2$ & 35 \\
\hline
\end{tabular}

electrical charge and are able to establish Coulombic interactions, which strongly influence the adsorption process. In the present case, the best results (high amount of Res adsorbed in the shorter time) were obtained at acidic $\mathrm{pH}$, for all fabrics (Table 1). For cotton and bamboo, there was no adsorption at $\mathrm{pH}=7$; this can be because the cellulosic fibers develop negative surface charge in that solution, repelling Res, which is similarly charged. As the degree of fiber ionization depends upon $\mathrm{H}^{+}$concentration on bulk solution, more acidic $\mathrm{pH}(5)$ neutralizes fiber-Res repulsion and facilitates the adsorption process [19]. Thus, at acidic $\mathrm{pH}, 51.2 \%$ $\left(35 \mathrm{mg} / \mathrm{g}_{\text {fiber }}\right)$ of Res was adsorbed by cotton and $28.1 \%$ $\left(19 \mathrm{mg} / \mathrm{g}_{\text {fiber }}\right)$ by bamboo in $15 \mathrm{~min}$. Takagai and co-workers [4] reported that $69.4 \%$ of Res was adsorbed by cotton, at acidic $\mathrm{pH}(2.6)$ and $18{ }^{\circ} \mathrm{C}$. Their work suggests that higher the temperature lower the extent of adsorption. So, the smaller adsorption obtained in this study can be due to the higher temperature $\left(20^{\circ} \mathrm{C}\right)$ and $\mathrm{pH}(5)$ used.

It is known that for protein based fibers, like silk, the acidic $\mathrm{pH}$ favors a cationic charged surface and, at the same time, improves Res adsorption [19]. Furthermore, silk can be considered highly functionalized, due to the amino and carboxylic groups in fiber composition, which can increase the probability of interactions between Res and silk and consequently, the amount of extract adsorbed [7]. As it was expected, the extent of adsorption observed for the combination Res-silk (Table 1) was higher than for Rescotton and Res-bamboo, at both $\mathrm{pHs}$ (more than $50 \%$ ).

It is important to refer that the initial concentration of Res used in the solution was most probably excessive, since the maximum amount adsorbed was $58.3 \%$ of that concentration. Hence, further studies should be carried out to obtain the optimal Res concentration, allowing the consequent reduction of this biofunctional fabric's cost.

Only fabrics obtained at $\mathrm{pH}=5$ were analyzed by FTIRATR (Figure 1), in order to confirm the presence of new interactions. The differences between the spectra of the textile material with and without adsorbed Res were not very notorious. Nevertheless in the Res-silk (Figure 1(D)) and Rescotton spectra (Figure 1(B)) it was possible to observe a slight decrease in the intensity of the band $3274 \mathrm{~cm}^{-1}$, which reflects the behavior of the broad intermolecular hydrogen bonded, O$\mathrm{H}$ stretch, present on the phenol groups of the extract [19]. This indicates that Res was adsorbed onto that fabric, corroborating the previous results (Table 1) where cotton and silk were the fibers to which Res had more affinity.

Fabrics, obtained at acid $\mathrm{pH}$, were also analyzed by spectrophotometric reflectance and the $K / S$ and $\Delta E$ values were determined (Table 2 ). The $K / S$ value is directly proportional to the extract concentration on the fabric, as postulated in the Kubelka-Munk law [19]. Furthermore, the $\Delta E$ values reflect the color difference between the fabric with and without Res and allow the confirmation of the $K / S$ results.

Based on the values present on Table 2, it is possible to 
A

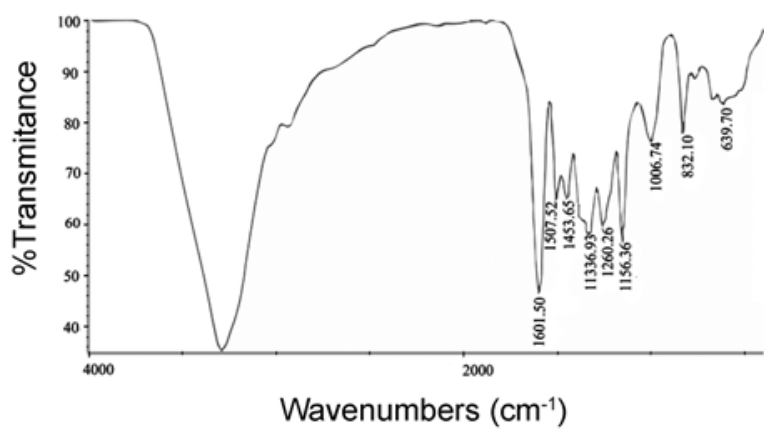

C

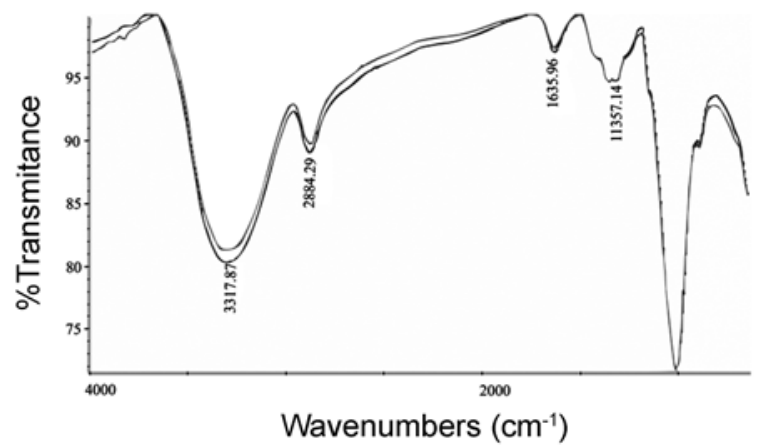

B

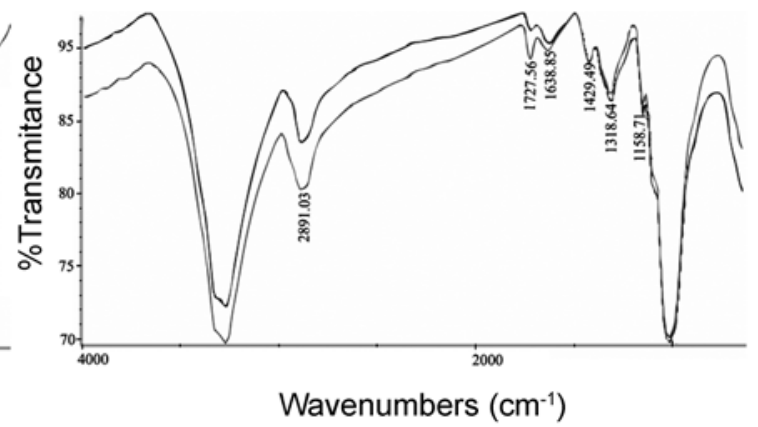

D

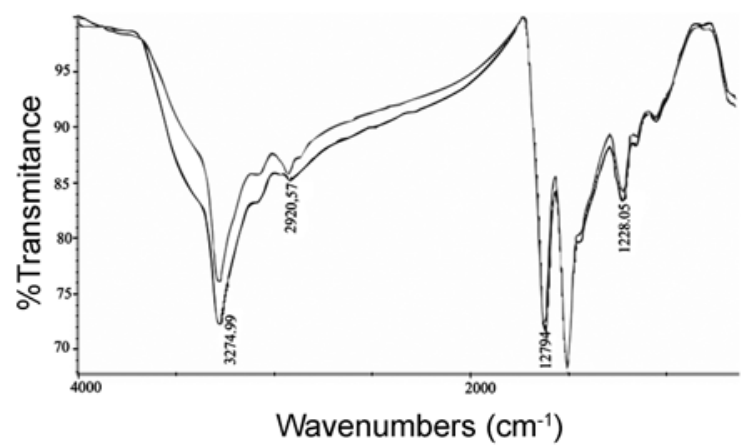

Figure 1. FTIR-ATR Spectra of the Res (KBr capsule) (A), the Res-cotton (B), Res-bamboo (C), and the Res-silk (D). The lither line is the fabric without Res and the darker line is the fabric with Res.

Table 2. $\Delta K / S$ values, color difference $(\Delta E)$, and images of the fabrics obtained at $\mathrm{pH}=5$

\begin{tabular}{lcccc}
\hline Fabric & $\Delta K / S$ & $\Delta E$ & Without Res & With Res \\
\hline & & & \\
Cotton & $1.70 \pm 0.05$ & $41.0 \pm 1.2$ & \\
\end{tabular}

Bamboo $0.90 \pm 0.0325 .2 \pm 0.75$

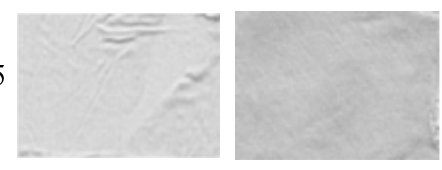

Silk

$4.00 \pm 0.012 \quad 42.7 \pm 1.3$

verify that Res was in fact present in all fabrics. Moreover, the $\Delta K / S$ and the $\Delta E$ were higher for the Res-silk combination. Therefore, Res had more affinity for silk than for cotton or bamboo, thus, corroborating the results obtained from the FTIR-ATR and the colorimetric analyses.

\section{Controlled Release Analysis}

To obtain an effectively biofunctional fabric it is necessary that the natural extract, with pharmacological properties, is released in a controlled manner, when in contact with skin [1]. Therefore, it is important to determine the amount of extract released from the fabric over time. As indicated, release tests were performed using the fabrics obtained from adsorption studies at $\mathrm{pH}=5$ and using three different solutions in order to mimic human body fluids: urine, acid sweat, and alkaline sweat. Water was used as control (Figure 2). Overall, it is possible to observe that Res was released in very small amounts (in all the solutions and for all the fabrics assayed). In Figure 2, a curve levelled is observed after $20 \mathrm{~min}$, which means that was achieved an equilibrium between the amount of Res in the solution and in the fabric.

Figure 2(A) shows that for cotton incorporating Res, after 60 min of immersion, only $0.5 \%$ of Res was released into water and just $1.0 \%$ was released in the acid sweat solution. Furthermore, the behavior of Res desorption from cotton was similar in urine and alkaline sweat like solutions and higher than in the other solutions $(4.7 \%$ in urine and $4.5 \%$ in the alkaline sweat). Res-cotton combination had the smallest and the highest released amounts. The lowest release was observed in water, about $1.0 \mathrm{ng} / \mathrm{ml}$, when compared with the amount released in the urine and alkaline acid sweat solutions $(8.0 \mathrm{ng} / \mathrm{m} l)$.

The amount of Res released from silk (Figure 2(C)) in alkaline solution was similar to the amount released from cotton. The profiles obtained for the other solutions were 

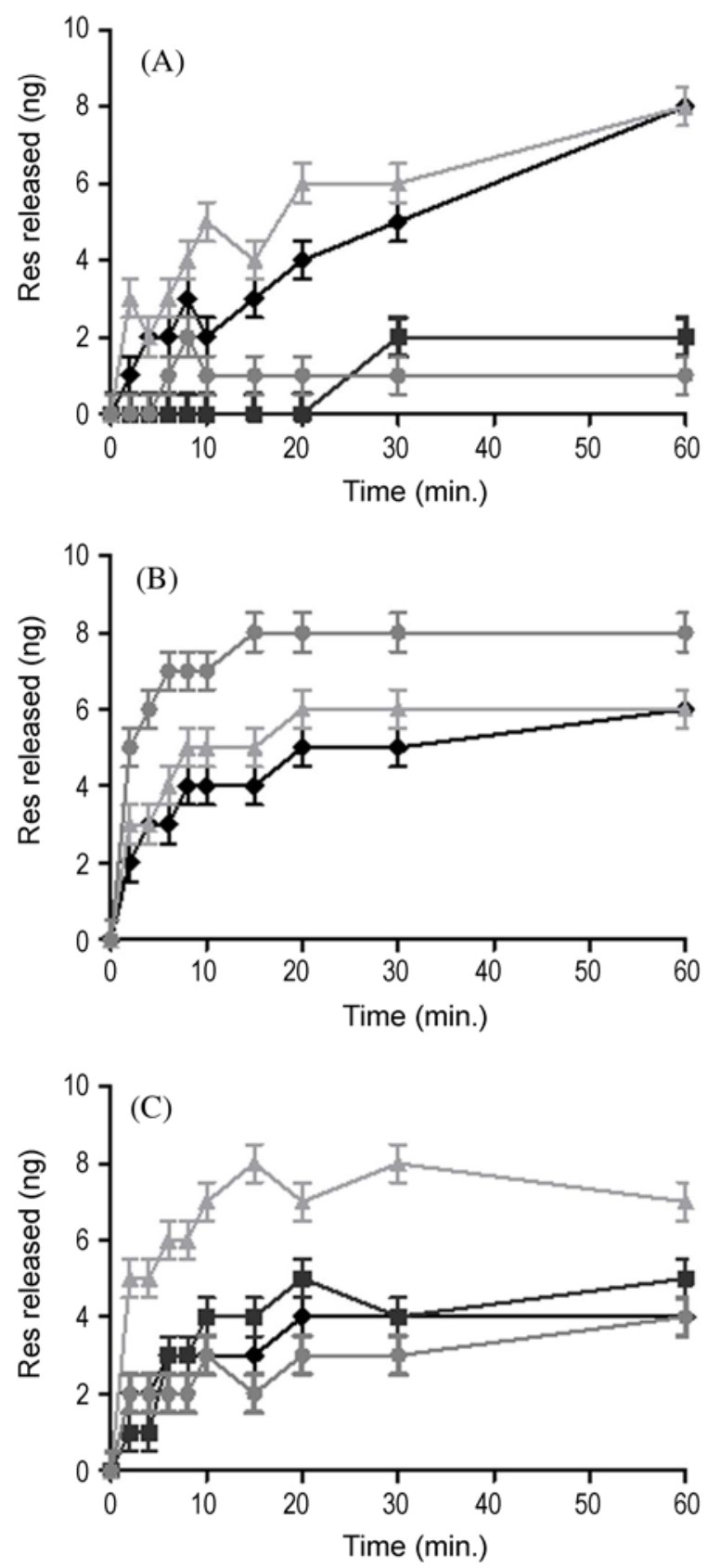

Figure 2. Controlled release graphics of the Res-cotton (A), the Res-bamboo (B), and the Res-silk (C) fabrics obtained at $\mathrm{pH}=5$. $(\diamond)$ in a urine like solution, $(\boldsymbol{\square})$ in a acid sweat like solution; ( $\Delta)$ in a alkaline sweat like solution; $(\mathbf{O})$ in water.

alike, with the lowest percentage of release being $2.1 \%$ in urine and $2.4 \%$ in water.

Res released from bamboo (Figure 2(B)) attained the highest amount, when compared to the other fabrics, with more than $5 \%$ of release $(8.7 \%$ in water, $5.7 \%$ in urine and $6.4 \%$ in alkaline sweat). Acid sweat assays were damaged and the results were not taken into consideration.

The adsorption and desorption are both related with the affinity of the extract to the fabric and dependent on the $\mathrm{pH}$ of the medium. For instance, at acid $\mathrm{pH}$ Res adsorbed to cotton at a high rate $(51.2 \%)$ and its release from this fabric, in acid sweat, was only $2.0 \mathrm{ng} / \mathrm{m} l$. Furthermore, at neutral $\mathrm{pH}$, the extract was adsorbed and in the controlled release assay at alkaline $\mathrm{pH} 8.0 \mathrm{ng} \cdot \mathrm{m} l^{-1}$ of Res was released. Both situations suggest that Res affinity to cotton varies with the solution $\mathrm{pH}$, being higher at acid $\mathrm{pH}$. Similarly, the adsorption on silk was higher at $\mathrm{pH}=5$ than $\mathrm{pH}=7$ and thus the release was lower in the solution of acid sweat, confirming that Res has more affinity to silk at acidic $\mathrm{pH}$.

Bamboo was the material displaying the lowest Res adsorption but also presented the lowest release, except in water. This contradiction may suggest that although Res establishes few interactions with bamboo, they are strong.

To authors' knowledge, there are no publications on desorption studies with this type of materials and in these conditions. However, desorption studies to assess the release of Res from cotton when immersed in organic solvents $(\mathrm{pH}=2.6)$ were made by Takagai and co-workers [4]. Their work suggested that $70 \sim 80 \%$ of Res was released in organic solvents and the temperature did not influence the desorption process.

Biofunctional fabrics have to be harmless to the skin cells. Therefore, it was necessary to assess the cytotoxicity of the fabrics incorporating Res. For that, fibroblast cells viability was evaluated by MTS test. This test measures the viability based on the conversion of MTS in a colored aqueous soluble formazan product by mitochondrial activity of viable cells. The amount of formazan produced by dehydrogenase enzymes is directly proportional to the number of living cells in the culture [20].

It was verified that the original textile (without Res) did not cause significant cell death $(5 \sim 20 \%)$, in opposition to the fabrics with Res. Res-silk was the combination promoting the lowest cell death (30\%), contrary to both cellulosic fabrics ( $75 \%$ for Res-cotton and $87 \%$ for Resbamboo) which presented high cytotoxicity. However, these discrepancy can not be directly explained by the results obtained of Res adsorbed or released (Table 1 and Figure 2). Moreover, the effect of Res was also assessed and it was verified that there was no interference of Res solution in the method used (data not shown).

Although these results showed that the amount of extract present in the fabrics is toxic for the animal cells, Aziz and co-workers [21] and Gescher and Steward [22] found no cytotoxicity when using a concentration of Res similar to the amount of Res released from fabrics assayed herein. Thus, this can indicate that is the Res, present in the fabric, which determines cytotoxicity and not the amount released.

\section{Conclusion}

The results obtained showed that it is possible to adsorb 
the natural resveratrol extract (Res) to natural fabrics, like cotton, bamboo and silk, attaining the highest efficiency at acidic $\mathrm{pH}$. Res showed more affinity to silk and less to bamboo. Moreover, the amount of Res released from these fabrics was minimal, for all the solutions assayed (watercontrol, acid and alkaline sweat, and urine).

Additionally, it was found that acidic $\mathrm{pH}$ favors the adsorption process. Concerning bamboo, it was possible to notice that although Res adsorption was low the resultant interaction is probably strong, as suggested by the small amount released.

Regarding cell interaction, it was possible to observe that the fabrics without extract did not show relevant toxicity, as it was expected. However, the fabrics with Res presented high cellular death, associating the presence of this compound to cytotoxicity. Although, the results did not allow the establishment of a relation between Res concentration and extension of cells death. Silk incorporating Res was the fabric that displayed the lowest cytotoxicity, pointing to a possible application as a biomaterial.

Therefore, this work can be considered the start point to create a new generation of fabrics made of natural materials and environmental friendly.

\section{References}

1. T. B. Volkmar in "Biofunctional Textiles and the Skin. Curr Probl Dermatol", 1st ed. (U. C. Hipler, P. Elsner, G. Burg, and P. H. Itin), Vol. 33, pp.51-66, Kanger Publishers, 2006.

2. K. Takai, T. Ohtsuka, Y. Senda, M. Nakao, K. Yamamoto, J. Matsuoka, and Y. Hirai, Microbio. Immuno., 46, 75 (2002).

3. P. A. Deshpande, M. B. Rao, and C. L. Rao, J. Appl. Polym. Sci., 76, 83 (2000).

4. Y. Takagai, T. Kubota, H. Kobayashi, T. Tashiro, A. Takahashi, and S. Igarashi, Anal. Sci., 21, 183 (2005).

5. R. Vieira, Master Dissertation, Universidade do Minho, Guimarães, 2006.

6. G. J. S. Alves, N. Raphaelli, and R. Fangueiro, in XXII
Congresso Nacional de Técnicos Têxteis - VIII Fenatêxtil, Recife, Brazil, 2006.

7. S. Hofmann, C. T. Wong Po Foo, F. Rossetti, M. Textor, G. Vunjak-Novakovic, D. L. Kaplan, H. P. Merkle, and L. Meinel, J. Control. Release, 111, 219 (2006).

8. G. H. Altman, F. Diaz, C. Jakuba, T. Calabro, R. L. Horan, J. Chen, H. Lu, J. Richmond, and D. L. Kaplan, Biomaterials, 24, 401 (2003).

9. M. S. Baliga and S. K. Katiyar, Photochem. Photobiol. Sci., 5, 243 (2006).

10. B. Yan, D. Xing, Y. Ding, J. Tao, and L. J. Du, J. Pharm. Biomed. Anal., 37, 297 (2005).

11. C. F. Hung, Y. K. Lin, Z. R. Huang, and J. Y. Fang, Biol. Pharm. Bull., 31, 955 (2008).

12. M.-T. Huang, G. Ghai, and C.-T. Ho, Comprehensive Reviews in Food Science and Food Safety, 3, 127 (2004).

13. M. H. Aziz, R. Kumar, and N. Ahmad, Int. J. Oncol., 23, 17 (2003).

14. E. Jr. Middleton, C. Kandaswami, and T. C. Theoharides, Pharmacol. Rev., 52, 673 (2000).

15. M. Araújo and E. M. Castro de Melo in "Manual de Engenharia Têxtil", 1st ed., vol. I, Fundação Calouste Gulbenkian, 1986.

16. ISC 114948-1, "International Standard - Urine - Absorbing Aids - Part 1: Whole-production Testing", 1996.

17. ISO105 E04, "Textiles - Tests for Colour Fastness - Part E04: Colour Fastness to Perspiration", 1997.

18. ISO 9073-8, "Textiles - Test Methods for Nonwovens Part 8: Determination of Liquid Strike-through Time (simulated urine)", 2000.

19. I. D. Ratter and M. M. Breuer in "The Physical Chemistry of Dye Adsorption", 1st ed., Academic Press Inc (London) LDT, 1974.

20. G. Malich, B. Markovic, and C. Winder, Toxicology, 124, 179 (1997).

21. M. H. Aziz, S. Reagan-Shaw, J. Wu, B. J. Longley, and N. Ahmad, FASEB J., 19, 1193 (2005).

22. J. A. Gescher and W. P. Steward, Cancer Epidem. Biomar., 12, 953 (2003). 